Books, videos, CD-ROMs, DVDs and any other relevant items submitted for a review in the $B D$ J should be addressed to: Kate Maynard, Assistant Editor, British Dental Journal, NPG, 4-6 Crinan Street, London N1 9XW

\section{MCQs for dentistry}

\section{K. F. M. Fan, J. Jones \\ UK: PasTest \\ price $€ 16.95$, pp 220 \\ ISBN 1904627528}

This book is published by PasTest which for many years has provided a range of study aids for undergraduate and postgraduate medical examinations. This particular title represents one of the company's first forays into the dental arena.

There is a helpful introduction which provides students with some tips on tackling multiple choice questions. The book comprises chapters on general dentistry, human disease, oral medicine, oral pathology, oral surgery, child dental health and orthodontics, therapeutics, dental materials, radiology and radiography and restorative dentistry. Each chapter has a number of MCQs of the conventional true/false variety. The answers to each question are on the following page together with some short notes which attempt to provide an explanation.

The production of the book presumably predates the updated guidance on prophylaxis of infective endocarditis and therefore does not reflect recent advice. However, there appears to be a misleading question in relation to rheumatic fever. Question 2.3 implies that all patients with a history of rheumatic fever develop valvular lesions, which is untrue. Thus a proportion of patients with a documented history of rheumatic fever do not have any valve lesion.

Reproduction of some images is not ideal, primarily due to the small size of some photographs, and this can lead to some confusion. At times some of the questions appear to be irrelevant to the section. Thus there is a section on the duties of a dental nurse in the chapter on radiology and radiography. In the chapter on oral surgery there is a question on burning mouth syndrome which leaves me struggling to think of a surgical approach to the management of this condition!
Previous publications from PasTest have generally focussed on a specific examination and therefore the target readership is obvious. In contrast, the target audience of this book is unclear although I suspect it will appeal to undergraduate students and postgraduates in the early years following qualification. This publication covers a number of topics examined in Parts A and B MFDS and as such I suspect that it will turn out to be a popular resource for candidates preparing for these examinations.

D. Felix

\section{Tooth erosion: prevention and treatment}

\author{
K. H. K. Yip, R. J. Smales, J. A. Kaidonis \\ India: Jaypee \\ price $\$ 35.00$, pp 130 \\ ISBN 8180617513
}

This book considers the aetiology, prevention and treatment of tooth erosion. In the preface the authors suggest that, whilst the prevalence of dental caries has reduced in many countries, the importance of erosion has increased, particularly since greater numbers of people are retaining teeth into old age. Research by this reviewer supports such a contention and has also shown that knowledge amongst the dental profession is poor regarding the recognition and management of erosion. With this in mind the publication of a carefully researched, succinctly written and well illustrated book on the topic is both timely and welcome. Five of the 11 chapters are presented as amended articles, the originals of which were published in different dental journals. However, the three editors were authors on four of these papers and the book is constructed so carefully that there is no suspicion of a cut and paste job.

The tone is set by Chapter 1 which considers the definitions, aetiology and appearances produced by different wear mechanisms in dentistry. The text is divided into short sections supported by 15 illustrations and 44 references, the latter indicated by unobtrusive superscript numbers that will not irritate the clinician and will be very useful to researchers and students who wish to investigate erosion further. This style is repeated throughout the book.

The dynamic nature of enamel and dentine and the critical role of saliva in maintaining the protection and normal functioning of the masticatory system are described in Chapter 2, which provides the basis upon which the management of erosion must be based. A thorough understanding of the interdependent physiological and psychological factors that may underlie erosion is essential. This is covered in Chapter 3 and carried on in Chapter 4 where prevention and control are considered, including the use of resinbased cements and glass-ionomers as physical barriers against acid attack. The important role of the dentist in diagnosing GERD is central to Chapter 5.

The next two chapters consider the diagnosis and control of intrinsic and extrinsic tooth erosion respectively. The use of microfine hybrid resin composites in association with acid etching or dentine bonding agents is recommended for advanced lesions. The need for restorative materials to be carefully selected in order to prevent further damage and destabilisation of the opposing dentition is covered in Chapter 8.

Chapter 9 deals with the two basic approaches to the oral rehabilitation of patients having extensive tooth tissue loss. A conformative approach is generally adopted when erosion is minimal, so that the occlusal vertical dimension (OVD) shows little or no alteration. More severe wear, perhaps associated with the loss of teeth, requires reorganisation of the occlusion with substantial modification of the existing occlusal jaw relationship. Case reports are shown in Chapter 10 and home care products for use in cases of erosion are considered in Chapter 11.

A book review can be a chore; this one was a joy. The authors are 


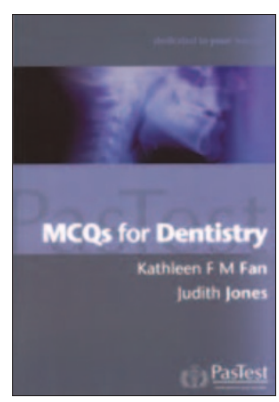

to be congratulated on producing a comprehensive account of a serious and increasingly common condition, in only 118 pages. The book reads well and is a visual delight: surely compulsory reading for dental practitioners.

W. P. Rock

\section{Evidence-based dentistry: an introduction}

\author{
A. K. Hackshaw, E. A. Paul, E. S. Davenport \\ UK: Blackwell Munksgaard \\ price €27.50, pp 225 \\ ISBN 1405124962
}

This book is designed as an introduction to understanding published research and its implications for the dental surgery. It is a ground-level book for those seeking to understand evidence-based dentistry and its significance for clinical practice.

There are 10 chapters in total. Chapter 1 summarises the purpose of evidencebased dentistry. Chapters 2 and 3 provide an introduction to some fundamental concepts used in the subsequent chapters. Chapter 4 uses these concepts to show how comparisons are made between groups of people. Chapters 2 , 5, 6 and 7 cover the main types of study design, namely: cross-sectional studies, randomised clinical trials, cohort studies and case-control studies. Chapter 8 compares and contrasts these different study designs. Chapter 9 discusses systematic reviews, while the last chapter summarises important statistical concepts.

Throughout the chapters, topical published papers from dental journals are used as worked examples. Each paragraph of the paper is numbered to allow the reader to pinpoint easily the particular section being discussed. Boxes are used extensively to present core information. Key points are summarised at the end of each chapter. These are followed by exercises with suggested answers.

The concept of intention-to-treat analysis has been clearly illustrated using
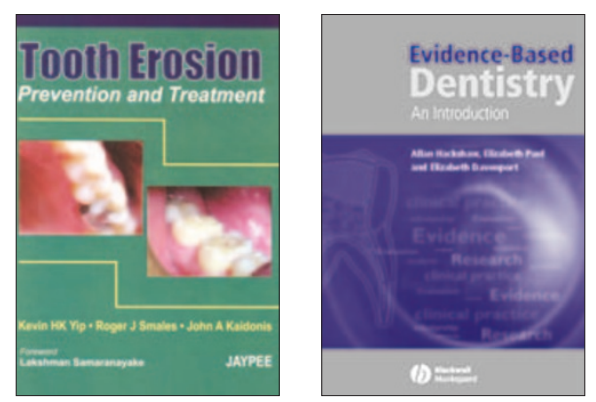

a hypothetical example, followed by an example taken from a dental journal. In the latter example, the analyses were reported to be intention-to-treat analyses, but they were actually per protocol analyses. The authors took the data given in the paper to re-calculate the correct figures, and used this example as an illustration of the method for intentionto-treat analysis.

Although guidelines for the appraisal of clinical trials and observational studies are provided with examples in appendices, there was no mention of allocation concealment (which is different from blinding) and the internationally recognised CONSORT (Consolidated Standards of Reporting Trials) statement.

The reading list at the end of the book proves to be up-to-date and useful for those wishing to pursue research methodology further.

In summary, I would recommend this book to dental undergraduates, postgraduates and dental practitioners. It is also a useful addition to any dental library, and the authors are to be congratulated.

C. A. Yeung 